\title{
61. Instantaneous Spectrograms of the Alkaline Earths by Disruptive Discharge.
}

\author{
By Hantaro Nagaoka, Daizo Nukiyama, and Tetsugoro Futagami. \\ Institute of Physical and Chemical Research.
}

(Rec. April 10, 1927. Comm. by H. NAGAOKA, M.I.A., April 12, 1927.)

The velocity of vapour and the interval of emission of spectral lines by electric discharge were first investigated by Schuster and Hemsalech ${ }^{1 /}$ with rapidly rotating film. We studied a large number of elements in a similar manner, but with a somewhat different object of examining the changes in the behaviour of the lines, such as reversal, broadening, and other characteristics, by following their course during a discharge. The present report is confined only to spectra of the alkaline earths.

The rotating film camera was provided with a drum, which was rotated by means of a small electric motor, making 5000 revolutions per minute, giving peripheral speed of $655^{\circ} \mathrm{cm} / \mathrm{sec}$ to the film, which had a diam. of $25 \mathrm{~cm}$. The camera was perforated by a slit, through which the spectra can be projected on the film. The spectrograph was provided with a Cornu prism, collimator and objective of quartz lens (aperture $30 \mathrm{~mm}$ and $50 \mathrm{~mm}$ resp.). Rough focussing for ultraviolet light was made by an uranium glass eye piece. For exact focussing repeated photographing on the rotating film was necessary. To obtain sufficient intensity of the light source, discharge from a condenser battery of 1.3 microfarad capacity was utilised. It was charged to about $25 \mathrm{~K}$.V. and discharged through a coil of $3.4 \times 10^{-5}$ henry, or only through a lead of $9.3 \times 10^{-6}$ henry selfinductance. This naturally led to electric oscillations, whose period was $4.7 \times 10^{-5}$ sec. and $2.2 \times 10^{-5}$ sec. resp. The discharge current calculated from the capacity and the charged voltage was $4.4 \mathrm{~K}$.A. and $9.4 \mathrm{~K}$.A. resp. The discharge with most elements was of the nature of electric explosion. The electrodes were metals of alkaline earths, and placed nearly in contact with each other. The discharge took place in air, but sometimes the electrodes

1) Schuster a. Hemsalech : Phil. Trans. A. 173 (1899) 189 ; Schenk; Astrophys. Journ. 14 (1901) 116 ; Royds ; Phil. Trans. A. 208 (1908) 333 ; Phil. Mag. (6) 19 (1910) 288. 
were enclosed in a closed vessel containing different gases, as the character of lines was sometimes affected by the nature of the surrounding medium. As our object was to discover the change in the emission of lines during the discharge, the length of the slit was made very short, so that it can be practically considered as a point; the rotating film was run parallel to the slit, and not perpendicular to it. When the distance between the electrodes was widened, the slit was made long, and the film run perpendicular to the slit. This process was confined to special lines only, whose character was desirable to examine more minutely.

All the spectrograms show the presence of regular electric oscillations, by which the lines are divided into a series of small sections, which mark the duration of $1.1 \times 10^{-5}$ sec. in the present report, and correspond to half period in the circuit, in which inductance coil is not included. This serves as time marks for estimating the interval of emission.

Numerous lines are emitted at the first half oscillation, and continue only for about half that period; some are excited little later during this stage, and requires close study. Unfortunately the aspect of these lines is difficult to reproduce in the photograms given in this paper. There are other lines, mostly belonging to different series, which continue to be emitted for $10^{-4}$ sec. or more. Some of these lines show remarkable changes during the emission; the broadening of the lines showing reversal is the most noteworthy point for spectroscopy.

For studying the reversals, it was desirable to get spectrograms in the ordinary manner and compare them with those obtained with rotating films. This was done simultaneously by means of another quartz spectrograph, to which the light emitted during the discharge was directed from the other side and photographed. The result was that the initial stage of the discharge was mostly imprinted on the spectrograms, so that the various stages of the changes to be next described were not to be traced on them.

Magnesium :-The most remarkable reversal is presented by the resonance line $2852(1 \mathrm{~S}-1 \mathrm{P})$. The line is reversed from the initial stage, and goes on broadening till it reaches the breadth of $60 \dot{\mathrm{A}}$. As the appearance of the line has to do with the valence electrons, it is probable that the broadening is connected with the formation of $\mathrm{MgO}$ covering the electrodes after the discharge. $3337,3332,3330(1 p-2 s)$ are initially faint, but become stronger; 2942, 2939, $2937(1 p-3 s)$ are very strong during the whole emission, and seem to be broadened to about $12 \dot{\mathrm{A}} ; 2782,2778,2776(1 p-4 s)$ show only weak reversal from the 
outset to the end. Triplet of diffuse series $3838,3832,3829(1 p-2 d)$ shows initial reversal, which is very fine, but it increases with time till the lines vanish; the second triplet 3097, 3093, $3091(1 p-3 d)$ are more sharply reversed as shown in the figure. The mean line $4481(2 \delta-3 \varphi)$ from ionised $\mathrm{Mg}$ is strongly emitted, and 2803, $2796(1 \sigma-1 \pi)$ show weak reversal.

Calcium :-The "flame line" $4227(1 \mathrm{~S}-1 \mathrm{P})$ is characterised by the strong reversal, which suddenly broadens to the extent of $220 \dot{A}$; this takes place near the closing stage and is very diffuse. Properly speaking, it may be due to other causes than that causing the reversal ; as it is a resonance line, the extraordinary broadening may be connected with the formation of $\mathrm{CaO}$, which usually covers the electrodes after the discharge in air or oxygen. The behaviour of this line is analogous to that of $\mathrm{Mg}$ 2852. The triplet 3285, 3274, $3269(1 p-4 s)$ shows faint reversal; the phenomenon is to be found in lines of the diffuse series $4455,4435,4426(1 p-2 d)$. 3644, 3631, $3624(1 p-3 d)$, 3362, $3350,3344(1 p-4 d), 3226,3215,3209(1 p-5 d)$. The lines 3737,3706 $(1 \pi-2 \sigma)$ are sharply emitted; but $\mathrm{H}(3968)$ and $\mathrm{K}$ (3934) of ionised $\mathrm{Ca}$ show strong and sharp reversal from the commencement to the end without variation. $3181,3179,3159$ belong to the same category and show similar reversal, although the life of the lines is short.

Strontium:-The line showing distinct reversal is $4078\left(1 \pi_{1}-1 \sigma\right)$ only; the other lines are strongly emitted and belong to ionised $\mathrm{Sr}$. They are $4305\left(1 \pi_{1}-2 \sigma\right), 4161\left(1 \pi_{2}-2 \sigma\right), 4216\left(1 \pi_{2}-1 \sigma\right) ; 3475\left(1 \pi_{1}-2 \delta^{\prime}\right)$, $3464\left(1 \pi_{1}-2 \delta\right), 3381\left(1 \pi_{2}-2 \delta\right)$.

Barium :- The strong lines in the barium spectrum consist of 4554 $\left(1 \pi_{1}-1 \sigma\right), 4934\left(1 \pi_{2}-1 \sigma\right), 4166\left(1 \pi_{1}-2 \delta^{\prime}\right), 4131\left(1 \pi_{1}-2 \delta\right), 3893\left(1 \pi_{2}-2 \delta\right)$ There are no lines showing distinct reversal.

It is to be remarked that on account of the small dispersion of the spectral apparatus used in obtaining the spectrograms, and the great pressure of the air at the instant of discharge, the partial vacuum following it, and the inrush of air into it, other effects are coming in to modify the spectra, so that no definite conclusion concerning the existence of reversal can be made. What were noted belong to those which can be detected with the spectrograph we have used. 


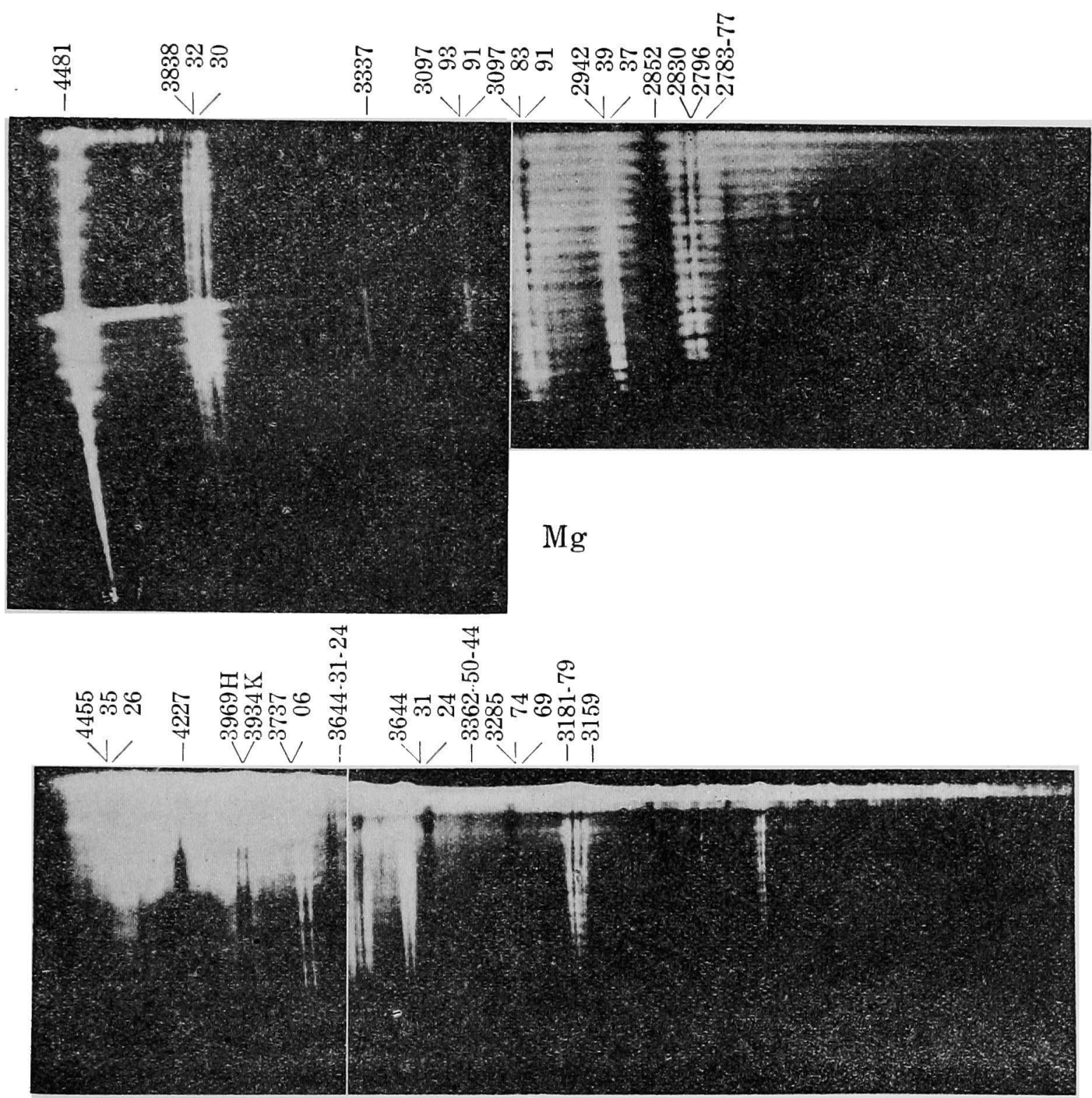

Ca

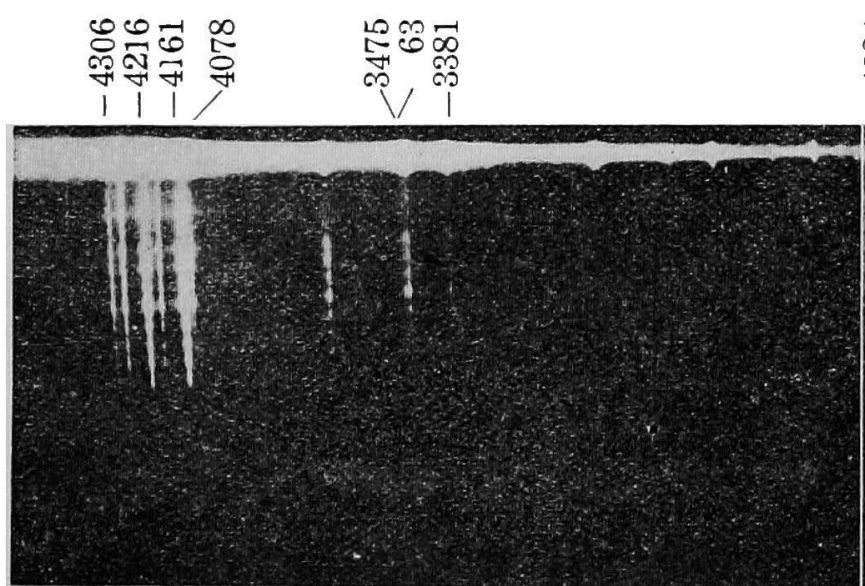

